\title{
TOXICIDADE DE AGROTÓXICOS USADOS NO CULTIVO DA CANA-DE-AÇÚCAR PARA FUNGOS ENTOMOPATOGÊNICOS NO SOLO
}

\author{
ALINE APARECIDA ALVES BOTELHO* \\ ANTONIO CARLOS MONTEIRO**
}

\begin{abstract}
Este trabalho teve por objetivo investigar se o tipo de solo influencia a toxicidade dos inseticidas aldicarbe e fipronil, dos herbicidas diuron e clomazone+ametrina e dos maturadores etil-trinexapac e sulfometurom-metílico, usados no cultivo da cana-de-açúcar para os fungos Metarhizium anisopliae e Beauveria bassiana. Os fungos foram inoculados em solo argiloso e arenoso, antes e após a adição dos produtos. Avaliou-se a sobrevivência depois de zero, um, quatro e sete dias de incubação. Aldicarbe reduziu a população dos fungos nos dois tipos de solo, com menor efeito tóxico no solo argiloso, enquanto fipronil pouco afetou a sobrevivência de $B$. bassiana em ambos os tipos de solo. Diuron mostrou menor efeito tóxico para $B$. bassiana, e, inversamente, maior efeito tóxico para $M$. anisopliae no solo arenoso. Clomazone+ametrina, etil-trinexapac e sulfometurommetílico reduziram a sobrevivência dos fungos nos dois tipos de solo, com menor efeito tóxico no solo argiloso. Os agrotóxicos utilizados no cultivo da cana-de-açúcar são tóxicos para $M$. anisopliae e $B$. bassiana, tanto em solo argiloso como arenoso. o efeito foi menor no solo argiloso e maior quando a adição dos agrotóxicos no solo ocorre logo antes ou após a inoculação dos fungos.
\end{abstract}

PALAVRAS-CHAVE: Metarhizium anisopliae; Beauveria bassiana; CONTROLE BIOLÓGICO; INSETICIDA; HERBICIDA; MATURADOR.

* Mestre em Microbiologia Agropecuária, ex-aluna do Programa de Pós-graduação em Microbiologia Agropecuária, Faculdade de Ciências Agrárias e Veterinárias (FCAV), Universidade Estadual Paulista (UNESP), Jaboticabal, SP (e-mail: botelho_alves@hotmail.com).

** Doutor em Ecologia e Recursos Naturais, Professor, Departamento de Produção Vegetal, FCAV/UNESP, Jaboticabal, SP (e-mail: montecar@fcav.unesp.br). 


\section{INTRODUÇÃO}

O solo constitui sistema vivo e os microrganismos desempenham papel importante na manutenção desse ecossistema, como componentes fundamentais das cadeias alimentares e dos ciclos biogeoquímicos. Entre suas atividades, incluem-se a formação e estruturação de solos, a decomposição da matéria orgânica, a ciclagem de nutrientes e a formação dos gases componentes da atmosfera terrestre (ZILLI et al., 2003).

O uso de grandes áreas e quantidades expressivas de insumos agrícolas pode afetar a qualidade do solo, sendo tais práticas questionadas pela comunidade científica quanto à sustentabilidade dos agroecossistemas. O termo qualidade do solo está relacionado às suas propriedades biológicas, físicas e químicas, essenciais para manter a produtividade agrícola a longo prazo com o mínimo possível de impacto negativo (TÓTOLA e CHAER, 2002).

O estado de São Paulo, maior produtor brasileiro de cana-de-açúcar, alcançou área total cultivada de 5,3 milhões de hectares na safra 2010/2011 (INPE, 2011). O uso de herbicidas de diferentes grupos químicos na cultura, tanto em pré como em pós-emergência, constitui prática comum no manejo das plantas daninhas presentes no canavial (COSTA et al., 2004). A aplicação de maturadores vegetais tem se tornado prática cada vez mais comum no setor sucroalcooleiro (VIANA et al., 2007), visando incrementar o teor de sacarose nos entrenós da cana, antecipar a maturação e aumentar a produtividade de açúcar (LEITE et al., 2009).

Para o controle de pragas usam-se inseticidas químicos. Contudo, a maioria dos insetos de importância para a cultura da cana-de-açúcar são pragas de solo como a cigarrinha da raiz Mahanarva fimbriolata Stal (1854) (Hemiptera: Cercopidae), o cerambicídeo da raiz Migdolus fryanus Westwood (1863) (Coleoptera: Vesperidae), a broca do rizoma Sphenophorus levis Vaurie (1978) (Coleoptera: Curculionidae) e larvas de escarabeídeos. Esses insetos são de difícil controle por meio de inseticidas químicos, o que abre oportunidade para a introdução de novos métodos (LEITE et al., 2003), como o uso de fungos entomopatogênicos, destacando-se o Metarhizium anisopliae (Metsch.) Sorokin (1879) capaz de promover o controle biológico de forma natural ou quando aplicado nos cultivos agrícolas (MOCHI, MONTEIRO e BARBOSA, 2005).

Trabalhos realizados com a adição de agrotóxicos ao meio de cultura sintético, usado para cultivar fungos entomopatogênicos, encontraram efeito tóxico (BATISTA FILHO, ALMEIDA e LAMAS, 2001; LOUREIRO et al., 2002). Usando tal metodologia, Botelho e Monteiro (2011) mostraram que inseticidas, herbicidas e maturadores usados no manejo da cana-de-açúcar exerceram efeito tóxico para M. anisopliae e Beauveria bassiana (Balsamo) Vuillemin (1912).

Os agrotóxicos chegam ao solo do ecossistema canavieiro pela deposição direta no momento da aplicação, através das águas das chuvas que os removem das partes áreas das plantas, ou pela incorporação da palhada após a colheita e podem afetar a sobrevivência de $M$. anisopliae e $B$. bassiana nesse ambiente, pois o solo é o reservatório natural dos fungos que infectam insetos (LANZA, MONTEIRO e MALHEIROS, 2004). Há vários tipos de solos, classificados de acordo com seus componentes. Alguns desses componentes têm a capacidade de adsorver os agrotóxicos retendo-os, o que pode dificultar sua distribuição e restringir sua ação tóxica (MOCHI, MONTEIRO e BARBOSA, 2005). Assim, o efeito tóxico para M. anisopliae e B. bassiana dos inseticidas, herbicidas e maturadores usados na cana-de-açúcar pode ser influenciado pelo tipo de solo.

Este trabalho teve por objetivo investigar se o tipo de solo influencia a toxicidade de inseticidas, herbicidas e maturadores usados no cultivo da cana-de-açúcar para os fungos entomopatogênicos M. anisopliae e B. bassiana.

\section{MATERIAL E MÉTODOS}

\subsection{FUNGOS E AGROTÓXICOS UTILIZADOS}

Foram utilizados os isolados E9 de $M$. anisopliae e IBCB66 de B. bassiana, mantidos na coleção do Laboratório de Microbiologia do Departamento de Produção Vegetal da Faculdade de 
Ciências Agrárias e Veterinárias da Universidade Estadual Paulista (FCAV-UNESP), Campus de Jaboticabal (SP). Os isolados foram cultivados em placas de Petri, contendo o meio de cultura de batata, dextrose e ágar (BDA), acondicionadas em estufa a $27 \pm 0,5^{\circ} \mathrm{C}$, em ausência de luz, durante 12 dias.

A partir de colônias crescidas nas placas foram preparadas suspensões de conídios em mistura (1:1) de solução de $\mathrm{NaCl}$ a $0,89 \%\left(p v^{-1}\right)$ e solução de Tween $80^{\circledR}$ a $0,1 \%\left(v v^{-1}\right)$. Após vigorosa agitação em agitador elétrico de tubos, as suspensões foram padronizadas na concentração de 2,7 x $10^{7}$ conídios $\mathrm{mL}^{-1}$, com auxílio da câmara de Neubauer.

Foram usados os seguintes agrotóxicos, descritos pelo ingrediente ativo, seguido pelo grupo químico e dose recomendada pelo fabricante: A) inseticida: fipronil (pirazol, $200 \mathrm{~g} \mathrm{ha}^{-1}$ ), aldicarbe (metilcarbamato oxima, 1,5 $\mathrm{kg} \mathrm{ha}^{-1}$ ); B) herbicida: diuron (ureia substituídas, 3,2 L ha-1), clomazone+ametrina (isoxazolidionas, 5,0 L ha-1); C) maturador: sulfometurommetílico (sulfonilureia, $20 \mathrm{~g} \mathrm{ha}^{-1}$ ), etil-trinexapac (dioxociclohexanocarboxilato de etila, 1,0 L ha-1) (COMPÊNDIO..., 1999). Essesagrotóxicosforamescolhidos porteremsidoclassificados comotóxicos aos fungos em experimentos laboratoriais conduzidos em meio de cultura (BOTELHO e MONTEIRO, 2011), exceto fipronil para $M$. anisopliae e foram preparados em volumes adequados aos ensaios.

\subsection{SOLO}

Foram utilizados dois tipos de solos, coletados na profundidade de 0 a $20 \mathrm{~cm}$ em matas de preservação ambiental, como segue: A) Latossolo vermelho textura argilosa (53\% de argila, $18 \%$ de silte e $29 \%$ de areia), coletado em propriedade agrícola no Distrito de Lusitânia, Município de Jaboticabal, SP $\left(21^{\circ} 07^{\prime} 04^{\prime \prime} \mathrm{S} ; 48^{\circ} 16^{\prime} 44^{\prime \prime} \mathrm{W}\right)$, com as seguintes características químicas: $\mathrm{pH}\left(\mathrm{CaCl}_{2}\right) 6,0$; matéria orgânica $=27 \mathrm{mmol}_{\mathrm{c}} \mathrm{dm}^{-3} ; \mathrm{P}$ (resina) $=24 \mathrm{mg} \mathrm{dm}^{-3} ; \mathrm{K}=4,4 \mathrm{mmol}_{\mathrm{c}} \mathrm{dm}^{-3}$; $\mathrm{Ca}=116 \mathrm{mmol}_{\mathrm{c}} \mathrm{dm}^{-3} ; \mathrm{Mg}=48 \mathrm{mmol}_{\mathrm{c}} \mathrm{dm}^{-3} ; \mathrm{H}+\mathrm{Al}=18 \mathrm{mmol}_{\mathrm{c}} \mathrm{dm}^{-3} ; \mathrm{SB}=168,5 \mathrm{mmol}_{\mathrm{c}} \mathrm{dm}^{-3}$; $\mathrm{T}=186,4 \mathrm{mmol}_{\mathrm{c}} \mathrm{dm}^{-3} ; \mathrm{V}=90 \%$; B) Argissolo vermelho amarelo textura arenosa média $(15 \%$ de argila, $7 \%$ de silte e $78 \%$ de areia), coletado em propriedade agrícola do Município de Monte Alto, SP $\left(21^{\circ} 21^{\prime} 02^{\prime \prime} \mathrm{S} ; 48^{\circ} 31^{\prime} 17^{\prime \prime} \mathrm{W}\right)$, apresentando as seguintes características químicas: $\mathrm{pH}\left(\mathrm{CaCl}_{2}\right) 5,0$; matéria orgânica $=27 \mathrm{mmol}_{\mathrm{c}} \mathrm{dm}^{-3} ; \mathrm{P}$ (resina) $=10 \mathrm{mg} \mathrm{dm}^{-3} ; \mathrm{K}=3,2 \mathrm{mmol}_{\mathrm{c}} \mathrm{dm}^{-3}$; $\mathrm{Ca}=35 \mathrm{mmol}_{\mathrm{c}} \mathrm{dm}^{-3} ; \mathrm{Mg}=12 \mathrm{mmol}_{\mathrm{c}} \mathrm{dm}^{-3} ; \mathrm{H}+\mathrm{Al}=47 \mathrm{mmol}_{\mathrm{c}} \mathrm{dm}^{-3} ; \mathrm{SB}=50,2 \mathrm{mmol}_{\mathrm{c}} \mathrm{dm}^{-3}$; $\mathrm{T}=97,2 \mathrm{mmol}_{\mathrm{c}} \mathrm{dm}^{-3} ; \mathrm{V}=52 \%$.

Os solos foram secos em temperatura ambiente, destorroados e peneirados em peneira com malha de $1 \mathrm{~mm}$. Em seguida, foram armazenados em sacos de plástico até a utilização. Determinouse a capacidade de saturação de água de cada tipo de solo antes da execução dos experimentos.

\subsection{MONTAGEM DOS ENSAIOS EXPERIMENTAIS E AVALIAÇÃO DA SOBREVIVÊNCIA DOS FUNGOS}

Para cada tratamento foram utilizadas quatro placas de Petri de $90 \mathrm{~mm}$ de diâmetro, contendo $80 \mathrm{~g}$ de solo. Na face interna da tampa de cada placa foram fixados, com fita adesiva, dois palitos de madeira para facilitar as trocas gasosas. As placas foram envolvidas por dupla camada de papel de embrulho e autoclavadas a $121^{\circ} \mathrm{C}$ e $1 \mathrm{Kgf} \mathrm{cm}^{-2}$ por $1 \mathrm{~h}$.

Os solos receberam água destilada estéril até atingir $65 \%$ da capacidade de saturação e permaneceram em repouso durante $1 \mathrm{~h}$ para estabilização. Em seguida, foram aplicados os fungos e agrotóxicos, constituindo os seguintes tratamentos: T1) adição de $2 \mathrm{~mL}$ de suspensão fúngica na superfície do solo e após $1 \mathrm{~h}$ adição do agrotóxico na superfície do solo; T2) adicionou-se o agrotóxico na superfície do solo e após $1 \mathrm{~h}$ foram acrescentados $2 \mathrm{~mL}$ de suspensão fúngica na superfície do solo; T3) adição de $2 \mathrm{~mL}$ de suspensão fúngica na superfície do solo e após $48 \mathrm{~h}$ adição do agrotóxico na superfície do solo. Para cada tratamento efetuou-se o controle, constituído da adição de $2 \mathrm{~mL}$ de suspensão fúngica e água destilada esterilizada na quantidade correspondente a cada agrotóxico. As placas foram pesadas e permaneceram em estufa a $27 \pm 0,5^{\circ} \mathrm{C}$, no escuro, até o final do ensaio.

Avaliou-se a sobrevivência dos fungos pela determinação do número de unidades formadoras de colônias (UFC grama de solo úmido-1) nos períodos de zero, 1, 4 e 7 dias de 
incubação. Antes da avaliação, as placas foram pesadas e a água perdida reposta usando-se água destilada esterilizada. Em seguida, amostra de 1,0 g de solo (composta de $15 \mathrm{sub}$ amostras de solo úmido) foi colhida na superfície de cada placa e suspendida em $9 \mathrm{~mL}$ de solução (1:1) de $\mathrm{NaCl}$ a $0,89 \%\left(\mathrm{p} \mathrm{v}^{-1}\right)$ e Tween $80^{\circledR}$ a $0,1 \%\left(\mathrm{v} \mathrm{v}^{-1}\right)$. A partir de diluição seriada adequada da suspensão foram semeados $0,1 \mathrm{~mL}$ em três placas de Petri, contendo o meio de Joussier e Catroux (1976), modificado pela supressão do suco de legumes e oxgall. Essas placas foram mantidas em estufa a $27 \pm 1^{\circ} \mathrm{C}$, no escuro, durante 5 dias.

\subsection{ANÁLISE ESTATÍSTICA}

Para cada tratamento foram feitas quatro repetições. Adotou-se o delineamento experimental inteiramente casualizado, sendo os dados submetidos à análise de variância pelo teste $\mathrm{F}$ e as médias comparadas pelo teste de Tukey a $5 \%$ de probabilidade. Para a execução das análises usou-se o programa ESTAT (1997).

\section{RESULTADOS E DISCUSSÃO}

O inseticida aldicarbe afetou a sobrevivência de $B$. bassiana em ambos os solos (Figuras $1 \mathrm{~A}$ e 1B). A sobrevivência do fungo foi menor no solo arenoso, principalmente quando a inoculação do fungo ocorreu uma hora antes (T1) ou uma hora após (T2) a adição do inseticida, sendo esses os tratamentos que mais afetaram a sobrevivência de $B$. bassiana nos dois tipos de solo. Esse resultado está congruente com o estudo de Santos e Monteiro (1994) que obtiveram drástica redução da população total de fungos do solo logo após a aplicação de aldicarbe. Usando meio de cultura, Tamai et al. (2002) verificaram que inseticidas pertencentes ao mesmo grupo químico mostraram-se muito tóxicos para $B$. bassiana.

A presença de fipronil pouco afetou a sobrevivência do fungo em ambos os tipos de solo. Apenas quando a inoculação no solo arenoso foi realizada uma hora após a adição do inseticida (T2) observou-se pequena redução da população de $B$. bassiana (Figuras $1 \mathrm{C}$ e $1 \mathrm{D}$ ). Almeida, Alves e Almeida (2000) aplicaram fipronil junto com B. bassiana no sulco de plantio da cana-deaçúcar e verificaram que houve aumento da eficiência do controle de $M$. fimbriolata. Afirmaram que essa pode ser eficiente estratégia no controle de pragas na cana-de-açúcar, não influenciando a ação do entomopatógeno.

A sobrevivência de $M$. anisopliae foi negativamente afetada pelo inseticida aldicarbe em ambos os solos (Figuras 2A e 2B). No solo argiloso, o efeito tóxico foi menor e não houve influência da forma de aplicação, pois não se verificou diferença significativa entre os tratamentos (T1, T2 e T3), apenas entre esses e a testemunha. No solo arenoso, o efeito tóxico do inseticida foi maior e reduziu significativamente a sobrevivência do fungo, notadamente nos tratamentos em que a inoculação ocorreu uma hora antes (T1) ou uma hora após (T2) a adição do inseticida.

A presença de diuron no solo influenciou a sobrevivência de $B$. bassiana, com menor efeito negativo no solo arenoso onde ocorreu pequena redução da população quando o fungo foi inoculado no solo uma hora após a adição do herbicida (T2) (Figura 3B). No solo argiloso, a ação tóxica foi maior havendo redução significativa da sobrevivência em todos os tratamentos, com destaque para o tratamento T2 em que ocorreu a menor população do fungo ao longo do período de avaliação (Figura 3A). Andaló et al. (2004) observaram redução do crescimento e produção de conídios de $B$. bassiana em experimentos in vitro realizados com os herbicidas diuron e glifosato.

O herbicida diuron, fortemente adsorvido pelos coloides de argila ou pela matéria orgânica do solo, pode ser lixiviado em solos arenosos (RODRIGUES e ALMEIDA, 2005). Uma das formas 
de reduzir sua concentração no solo é a degradação microbiana realizada por fungos e bactérias (CASTILLO et al., 2006). B. bassiana mostrou-se eficiente degradador de diuron, embora as transformações do herbicida realizadas pelo fungo sejam, na maioria das vezes, incompletas (TIXIER et al., 2000). Neste trabalho, a maior sobrevivência de B. bassiana foi obtida no solo arenoso.
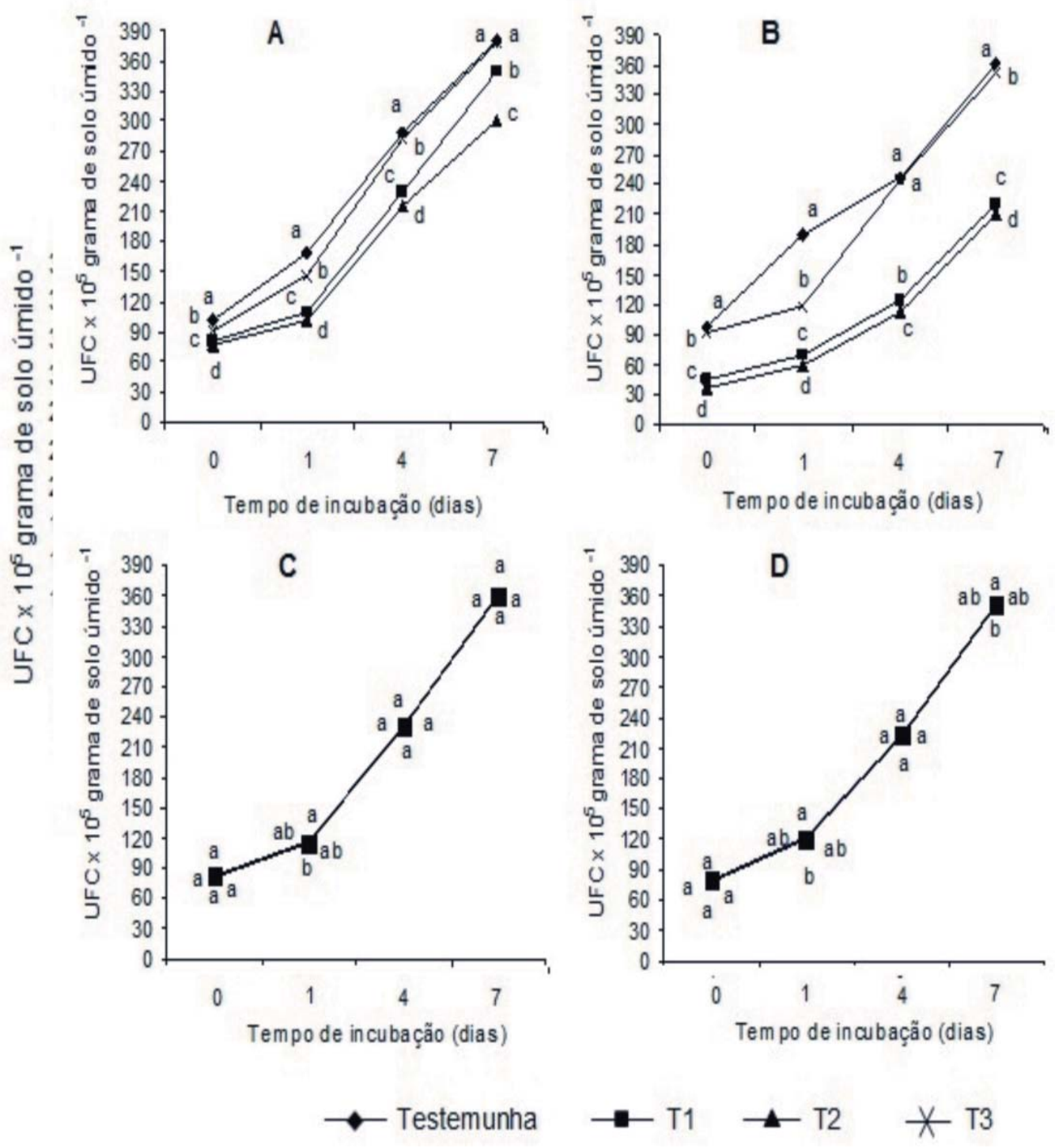
FIGURA 1 - SOBREVIVÊNCIA DE Beauveria bassiana INOCULADO EM SOLO ARGILOSO E SOLO ARENOSO ADICIONADOS DOS INSETICIDAS QUÍMICOS ALDICARBE (A E B, RESPECTIVAMENTE) E FIPRONIL (C E D, RESPECTIVAMENTE), NAS SEGUINTES FORMAS DE APLICAÇÃO: T1 - INOCULAÇÃO DO FUNGO NO SOLO 1 HORA ANTES DA APLICAÇÃO DO INSETICIDA; T2 - INOCULAÇÃO DO FUNGO NO SOLO 1 HORA APÓS A APLICAÇÃO DO INSETICIDA; T3 - INOCULAÇÃO DO FUNGO NO SOLO 48 HORAS APÓS A

\section{APLICAÇÃO DO INSETICIDA}


O efeito de clomazone+ametrina mostrou-se praticamente inverso ao do herbicida anterior. No solo argiloso, houve pequena diminuição da sobrevivência de $B$. bassiana, enquanto no solo arenoso verificou-se acentuada redução da população do fungo (Figuras 3C e 3D). Tal resultado deveu-se, possivelmente, ao pouco contato do herbicida com o entomopatógeno no solo argiloso, pois segundo Cumming, Doyle e Brown (2002), o agrotóxico fica adsorvido na superfície dos coloides desse solo. Em ambos os solos, os tratamentos com a inoculação do fungo uma hora antes (T1) ou uma hora após (T2) a adição do herbicida foram os que mais afetaram a sobrevivência de $B$. bassiana.
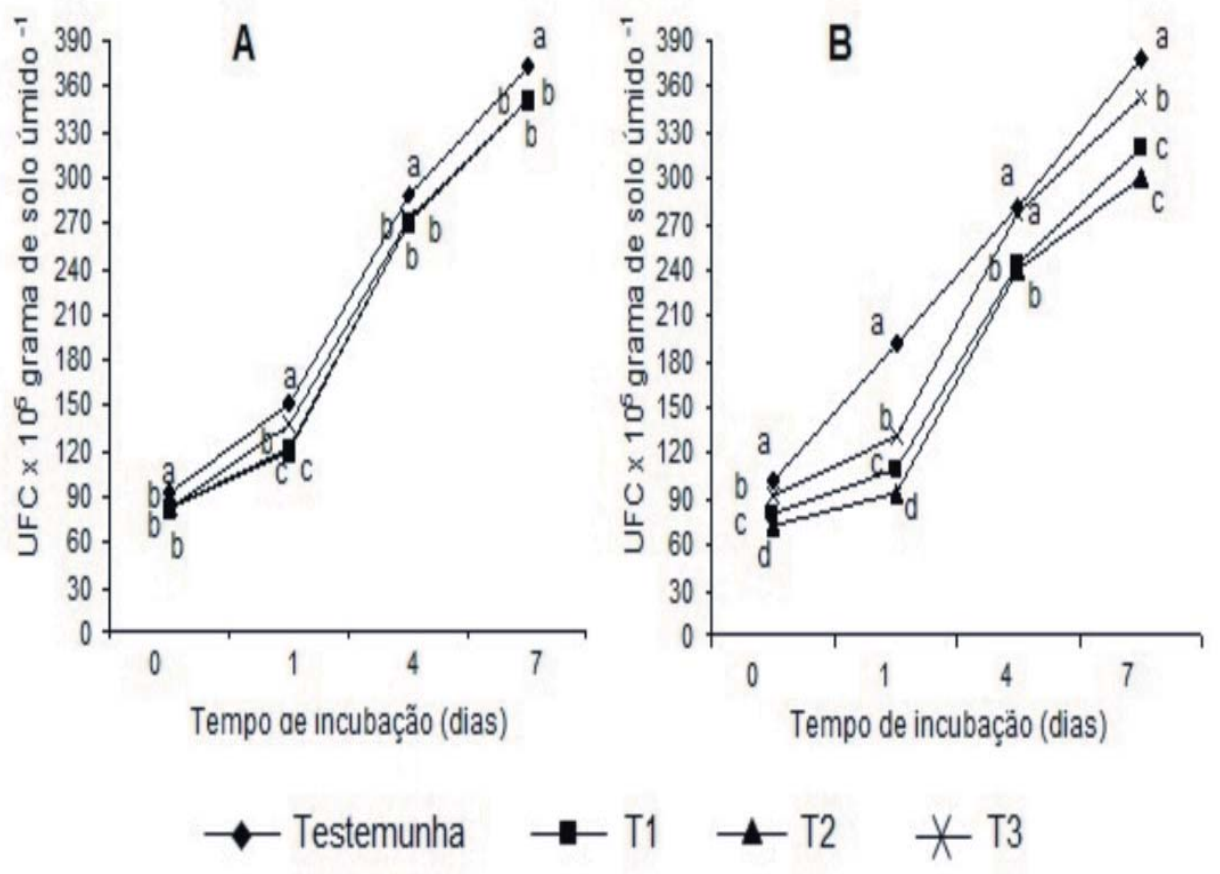

FIGURA 2 - SOBREVIVÊNCIA DE Metarhizium anisopliae INOCULADO EM SOLO ARGILOSO (A) E SOLO ARENOSO (B) ADICIONADOS DO INSETICIDA QUÍMICO ALDICARBE, NAS SEGUINTES FORMAS DE APLICAÇÃO:
T1 - INOCULAÇÃO DO FUNGO NO SOLO 1 HORA ANTES DA
APLICAÇÃO DO INSETICIDA; T2 - INOCULAÇÃO DO FUNGO NO
SOLO 1 HORA APÓS A APLICAÇÃO DO INSETICIDA;
T3 - INOCULAÇÃO DO FUNGO NO SOLO 48 HORAS APÓS A APLICAÇÃO DO INSETICIDA

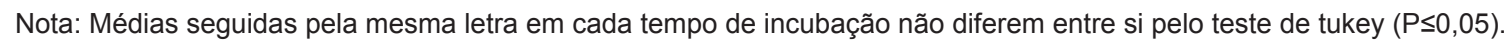

M. anisopliae mostrou-se bastante susceptível a ação tóxica dos herbicidas no solo (Figura 4). Diuron e clomazone+ametrina afetaram a sobrevivência do fungo nos dois tipos de solo, mas principalmente no arenoso em que ocorreram as maiores reduções da população do fungo (Figuras 4B e 4D). No solo argiloso, o efeito tóxico foi menor (Figuras 4A e 4C) e em ambos os solos verificaram-se os maiores efeitos quando o fungo foi inoculado uma hora antes (T1) ou uma hora após (T2) a adição dos herbicidas.

Os maturadores químicos adicionados ao solo tiveram efeito negativo na sobrevivência de B. bassiana (Figura 5). Sulfometurom metílico reduziu acentuadamente a população do fungo 
no solo arenoso, quando a inoculação ocorreu uma hora antes (T1) ou uma hora após (T2) a adição do maturador (Figura 5B). No solo argiloso houve menor efeito tóxico sobre o fungo e após sete dias de incubação, apenas no tratamento T2 ocorreu acentuada redução da sobrevivência (Figura 5A). Efeito semelhante foi observado com a adição ao solo de etil-trinexapac que reduziu substancialmente a sobrevivência de $B$. bassiana nos tratamentos T1 e T2 no solo arenoso (Figura 5D) e também afetou negativamente a população no solo argiloso, nas três formas de aplicação, mas ocasionando menor redução da população do fungo (Figura 5C).
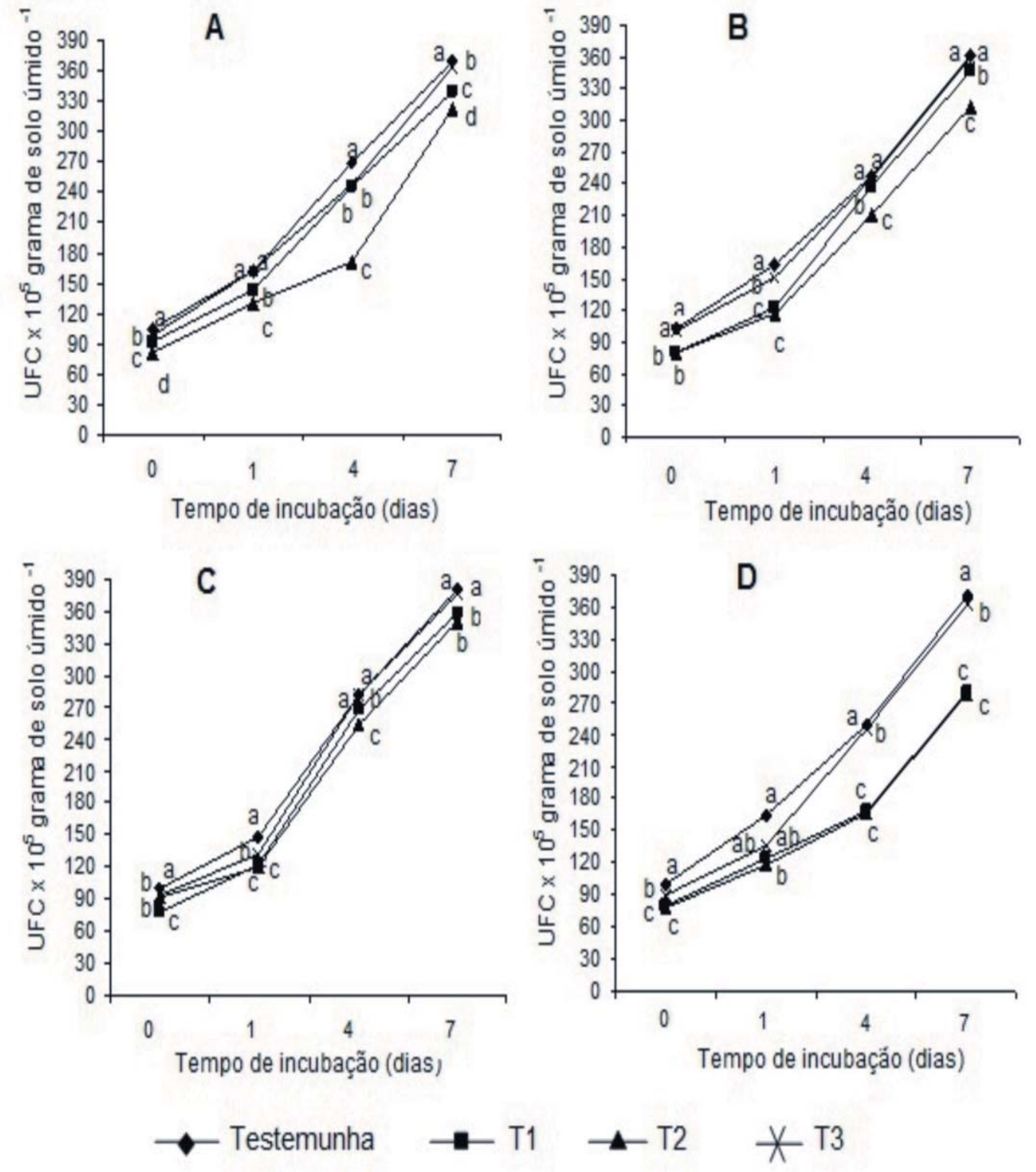

FIGURA 3 - SOBREVIVÊNCIA DE Beauveria bassiana INOCULADO EM SOLO ARGILOSO E SOLO ARENOSO ADICIONADOS DOS HERBICIDAS QUÍMICOS DIURON (A E B, RESPECTIVAMENTE) E CLOMAZONE+AMETRINA (C E D, RESPECTIVAMENTE), NAS SEGUINTES FORMAS DE APLICAÇÃO:

T1 - INOCULAÇÃO DO FUNGO NO SOLO 1 HORA ANTES DA APLICAÇÃO DO HERBICIDA QUÍMICO; T2 - INOCULAÇÃO DO FUNGO NO SOLO 1 HORA APÓS A APLICAÇÃO DO HERBICIDA QUÍMICO; T3 - INOCULAÇÃO DO FUNGO NO SOLO 48 HORAS APÓS A APLICAÇÃO DO HERBICIDA QUÍMICO

Nota: Médias seguidas pela mesma letra em cada tempo de incubação não diferem entre si pelo teste de tukey $(P \leq 0,05)$. 
Verificou-se ação similar dos maturadores para M. anisopliae e B. bassiana no solo. $O$ efeito causado por sulfometurom metílico nos solos arenoso e argiloso (Figuras 6A e 6B) e por etil-trinexapac no solo argiloso (Figura $6 \mathrm{C}$ ) assemelhou-se ao observado para $B$. bassiana nos respectivos solos. Contudo, a adição de etil-trinexapac no solo arenoso não reduziu a população de $M$. anisopliae em nenhum dos tratamentos, após sete dias de incubação (Figura 6D).
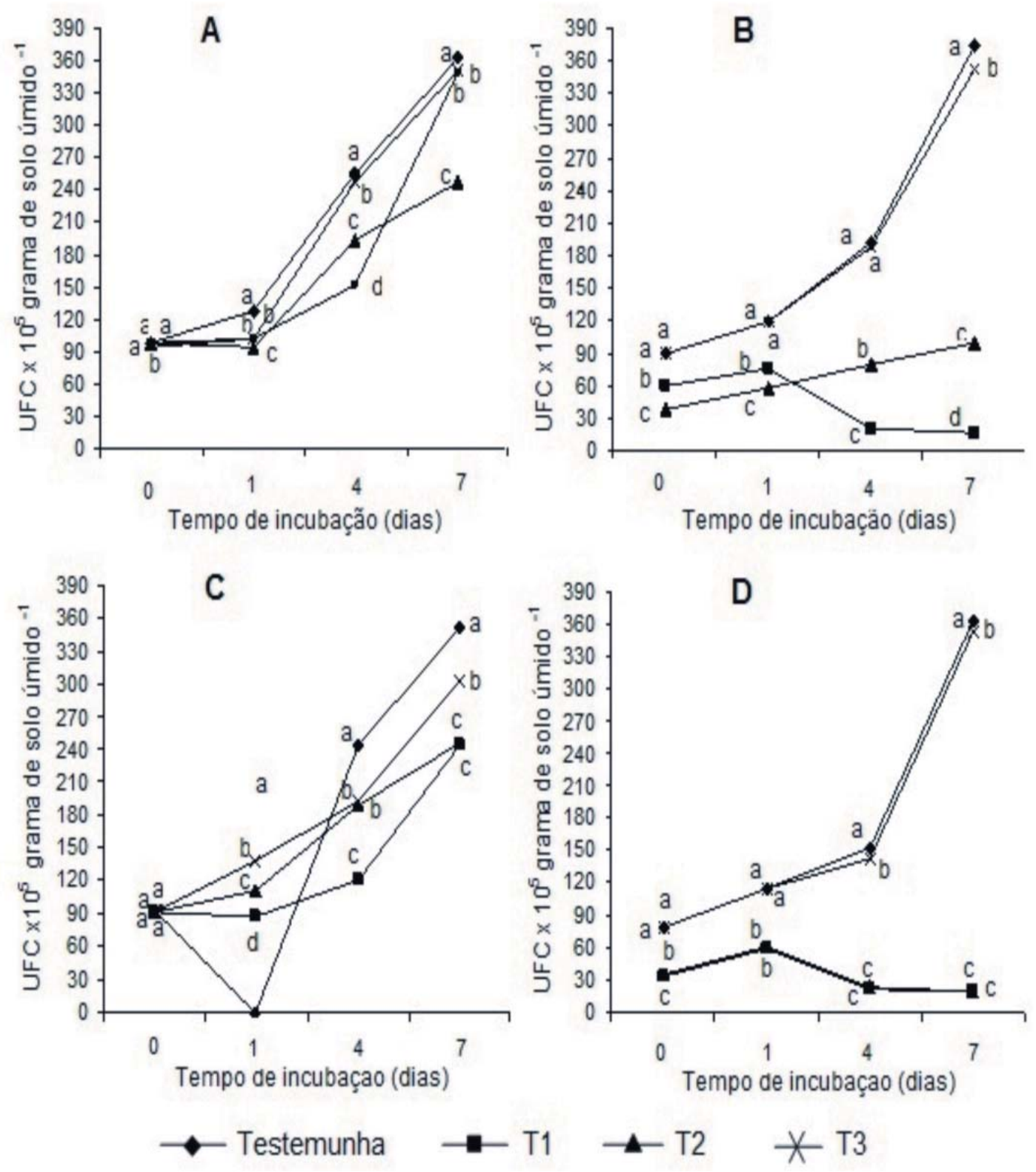

FIGURA 4 - SOBREVIVÊNCIA DE Metarhizium anisopliae INOCULADO EM SOLO ARGILOSO E SOLO ARENOSO ADICIONADOS DOS HERBICIDAS

QUÍMICOS DIURON (A E B, RESPECTIVAMENTE) E CLOMAZONE+ AMETRINA (C E D, RESPECTIVAMENTE) NAS SEGUINTES FORMAS DE APLICAÇÃO: T1 - INOCULAÇÃO DO FUNGO NO SOLO 1 HORA ANTES DA APLICAÇÃO DO HERBICIDA; T2 - INOCULAÇÃO DO FUNGO NO SOLO 1 HORA APÓS A APLICAÇÃO DO HERBICIDA; T3 - INOCULAÇÃO DO FUNGO NO SOLO 48 HORAS APÓS A APLICAÇÃO DO HERBICIDA 

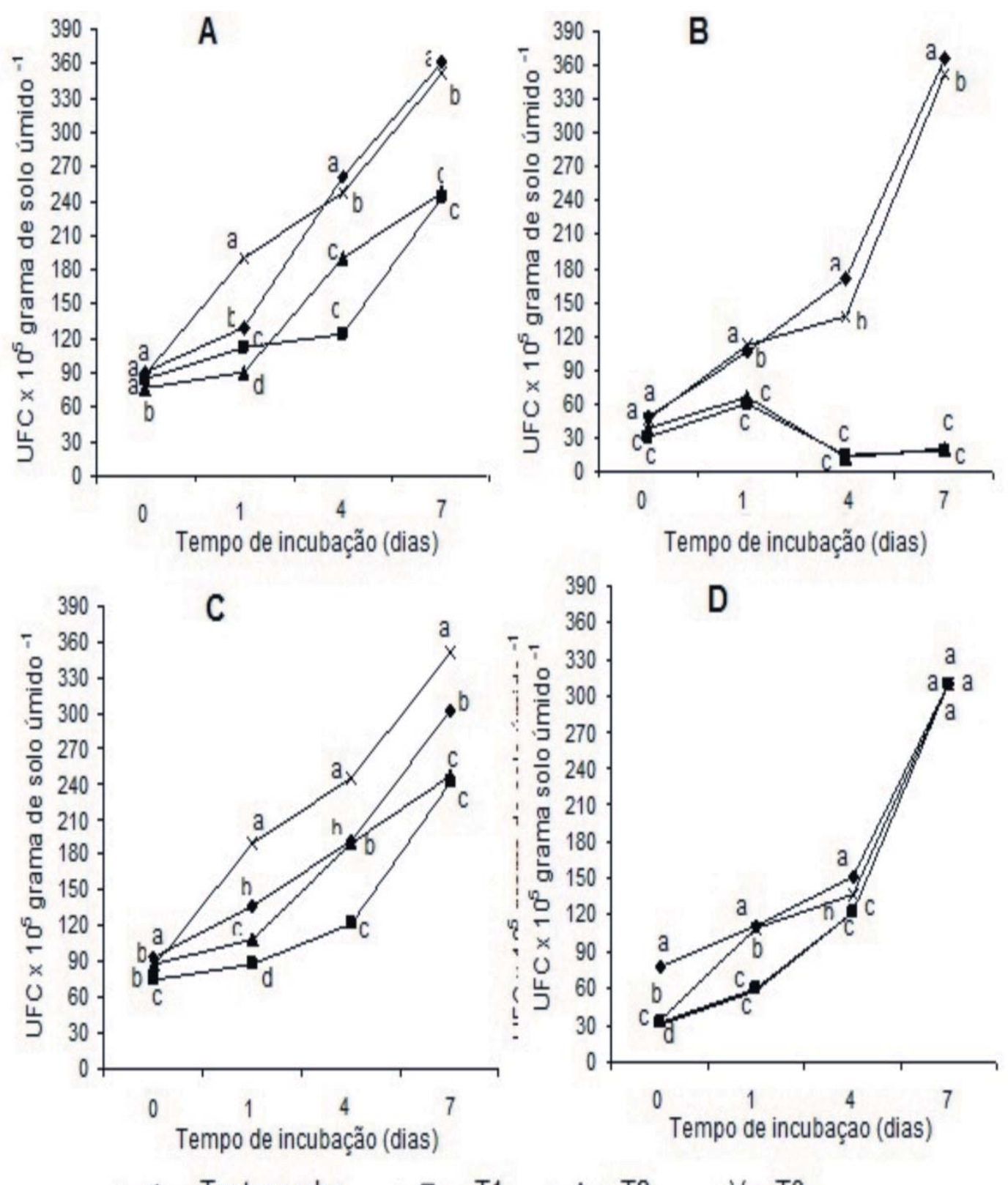

$\bullet$ Testemunha $\rightarrow-\mathrm{T} 1 \quad \bullet-\mathrm{T} 2 \quad$ * T3

FIGURA 5 - SOBREVIVÊNCIA DE Beauveria bassiana INOCULADO EM SOLO ARGILOSO E SOLO ARENOSO ADICIONADOS DOS MATURADORES QUÍMICOS SULFOMETUROM METÍLICO (A E B, RESPECTIVAMENTE)

E ETIL-TRINEXAPAC (C E D, RESPECTIVAMENTE), NAS SEGUINTES FORMAS DE APLICAÇÃO: T1 - INOCULAÇÃO DO FUNGO NO SOLO 1 HORA ANTES DA APLICAÇÃO DO MATURADOR; T2 - INOCULAÇÃO DO FUNGO NO SOLO 1 HORA APÓS A APLICAÇÃO DO MATURADOR; T3 - INOCULAÇÃO DO FUNGO NO SOLO 48 HORAS APÓS A APLICAÇÃO DO MATURADOR

Nota: Médias seguidas pela mesma letra em cada tempo de incubação não diferem entre si pelo teste de tukey $(P \leq 0,05)$.

Raríssimos são os trabalhos existentes na literatura sobre a ação de inseticidas e herbicidas para fungos entomopatogênicos no solo, e não foram encontrados estudos envolvendo maturadores. Pelos resultados desta investigação foi possível verificar que, a exceção do efeito do inseticida 
fipronil sobre $B$. bassiana nos dois tipos de solo, a ação tóxica dos produtos reduziu a sobrevivência de $M$. anisopliae e $B$. bassiana, tanto no solo argiloso como no arenoso. A sensibibilidade dos entomopatógenos aos agrotóxicos variou bastante em ambos os solos, sugerindo que o ambiente edáfico possa interferir na ação tóxica. Em experimentos laboratoriais conduzidos em meio de cultura, os agrotóxicos utilizados neste trabalho foram classificados como tóxicos aos fungos (BOTELHO e MONTEIRO, 2011), mas no solo essa toxicidade foi bastante atenuada.
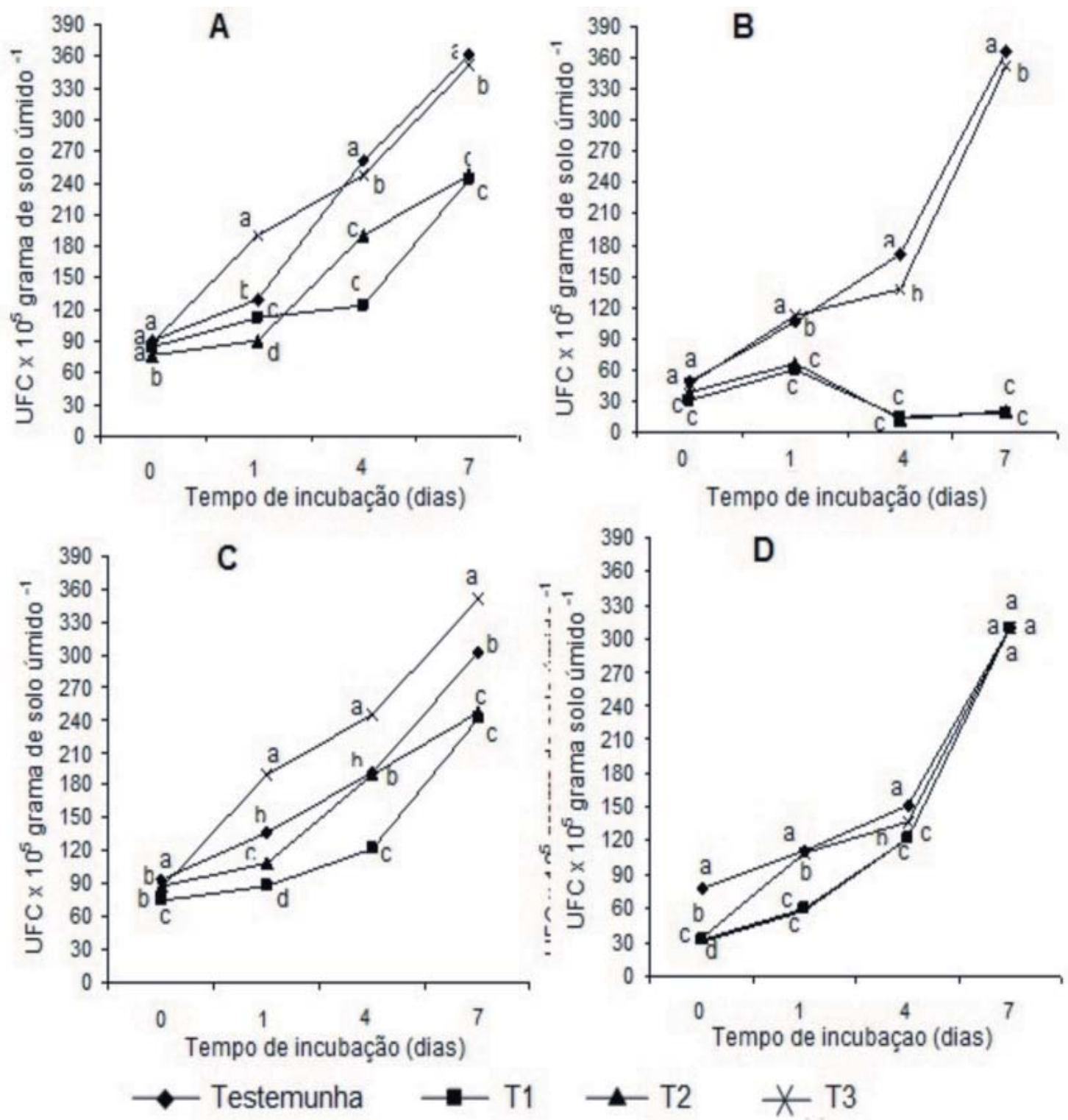

FIGURA 6 - SOBREVIVÊNCIA DE Metarhizium anisopliae INOCULADO EM SOLO ARGILOSO E SOLO ARENOSO ADICIONADOS DOS MATURADORES QUÍMICOS SULFOMETUROM METÍLICO (A E B, RESPECTIVAMENTE) E ETIL-TRINEXAPAC (C E D, RESPECTIVAMENTE), NAS SEGUINTES FORMAS DE APLICAÇÃO: T1 - INOCULAÇÃO DO FUNGO NO SOLO 1 HORA ANTES DA APLICAÇÃO DO MATURADOR; T2 - INOCULAÇÃO DO FUNGO NO SOLO 1 HORA APÓS A APLICAÇÃO DO MATURADOR; T3 - INOCULAÇÃO DO FUNGO NO SOLO 48 HORAS APÓS A APLICAÇÃO DO MATURADOR 
De acordo com Lanza, Monteiro e Malheiros (2009), o tipo de solo influencia a sobrevivência de fungos entomopatogênicos. Este estudo mostrou que também teve influência na ação tóxica dos agrotóxicos para os fungos. Na maioria das vezes, a sobrevivência foi maior no solo argiloso. $\mathrm{O}$ maior conteúdo de argila desse solo, aliado à presença de matéria orgânica que têm capacidade de adsorver substâncias químicas, provavelmente causaram a retenção de moléculas dos agrotóxicos reduzindo o efeito tóxico sobre os fungos. Além disso, a porosidade do solo arenoso certamente facilita a migração e distribuição dos produtos, favorecendo a ação tóxica.

O efeito tóxico foi maior quando os fungos foram aplicados no solo uma hora antes ou depois da adição dos agrotóxicos, situações em que ficaram por maior tempo expostos aos produtos. Esse fato revela que os entomopatógenos devem ser aplicados nos canaviais em épocas mais distintas possíveis das usadas para aplicar os agrotóxicos para não comprometer sua ação no controle de pragas da cana-de-açúcar.

\section{CONCLUSÂO}

Os inseticidas, herbicidas e maturadores utilizados no manejo da cultura da cana-de-açúcar são tóxicos para $M$. anisopliae e $B$. bassiana, tanto no solo argiloso como no arenoso. A toxicidade mostrou-se menor no solo argiloso do que no solo arenoso e o efeito tóxico foi maior quando os agrotóxicos foram adicionados logo antes ou após a inoculação dos fungos em ambos os solos.

\section{ABSTRACT \\ TOXICITY OF PESTICIDES USED IN SUGARCANE CULTIVATION AGAINST ENTOMOPATHOGENIC FUNGI IN SOIL}

This research aimed to investigate if the type of soil can influence the toxicity of the insecticides aldicarb and fipronil, the herbicides diuron and clomazone+ametryne and the ripeners ethyl trinexapac and sulfometurommethyl used in the cultivation of sugarcane, against the fungi Metarhizium anisopliae and Beauveria bassiana. The fungi were inoculated in clay and sandy soil, before and after the addition of pesticides. Survival was evaluated after zero, one, four and seven days of incubation. Aldicarb reduced the survival of $M$. anisopliae and $B$. bassiana in both soil types, with less toxic effect on the clay soil, while fipronil did not affect significantly $B$. bassiana survival on both soil types. Diuron had lower toxic effect over $B$. bassiana, and higher toxic effect on $M$. anisopliae in sandy soil. Clomazone+ametryne, ethyl trinexapac and sulfometurom-methyl reduced the survival of the fungi in both soil types, with less toxic effect on clay soil. The pesticides used in the cultivation of sugarcane are toxic to $M$. anisopliae and $B$. bassiana both in clay and sandy soil. Effect was lower in clay soil and higher when pesticides were added in the soil just before or after fungi inoculation.

KEY-WORDS: Metarhizium anisopliae; Beauveria bassiana; BIOLOGICAL CONTROL; INSECTICIDE; HERBICIDE; RIPENER.

\section{REFERÊNCIAS}

1 ALMEIDA, J.E.M.; ALVES, S.B.; ALMEIDA, L.C. Controle de Heterotermes tenuis (Hagen) (Isoptera: Rhinotermitidae) e Cornitermes cumulans (Kollar) (Isoptera: Termitidae) com inseticida fipronil associado ao fungo entomopatogênico Beauveria bassiana (Bals.) Vuill. em isca atrativa na cultura da cana-deaçúcar (Saccharum officinarum ). Arquivos do Instituto Biológico, São Paulo, v. 67, n. 2, p. 235-241, 2000.

2 ANDALÓ, V.; MOINO JUNIOR, A.; SANTA-CECÍLIA, L.V.C.; SOUZA, G.C. Compatibility of Beauveria bassiana with chemical pesticides for the control of the coffee root mealybug Dysmicoccus texensis Tinsley (Hemiptera: Pseudococcidae). Neotropical Entomology, v. 33, n.4, p. 463-467, 2004.

3 BATISTA FILHO, A.; ALMEIDA, J.E.M.; LAMAS, C. Effect of thiamethoxam on entomopathogenic microorganisms. Neotropical Entomology, v. 30, n.3, p. 437-447, 2001.

4 BOTELHO, A.A.A.; MONTEIRO, A.C. Sensibilidade de fungos entomopatogênicos a agroquímicos usados no manejo da cana-de-açúcar. Bragantia, Campinas, v. 70, n. 2, p. 361-369, 2011. 
5 CASTILLO, M.A.; FELIS, N.; ARAGON, P.; CUESTA, G.; SABATER, C. Biodegradation of the herbicide diuron by streptomyces isolated from soil. International Biodeterioration and Biodegradation, v. 58, n. 3-4, p. 196-202, 2006.

6 COMPÊNDIO de defensivos agrícolas: guia prático de produtos fitossanitários para uso agrícola. 6. ed. São Paulo: Andrei, 1999. 672 p.

7 COSTA. E.A.D.; MATALLO, M.B.; ALMEIDA, J.E.M.; LOUREIRO, E. S.; SANO, A.H. Efeito de herbicidas utilizados em cana-de-açúcar no desenvolvimento in vitro do fungo entomopatogênico Metarhizium anisopliae (Metsch.) Sorokin. Pesticidas: revista de ecotoxicologia e meio ambiente, v. 14, p. 19-24, 2004.

8 CUMMING, J. P.; DOYLE, R.B.; BROWN, P.H. Clomazone dissipation in four Tasmanian topsoils. Weed Science, v. 50, n. 3, p. 405-409, 2002.

9 ESTAT: sistema para análises estatísticas. Versão 2.0. Jaboticabal: Faculdade de Ciências Agrárias e Veterinárias, 1997 Disponível em: <http://www.fcav.unesp.br/download2/softwares/estat/>. Acesso em: 7 abr. 2010.

10 INPE. Instituto Nacional de Pesquisas Espaciais. Projeto Canasat, 2011. Disponível em: <http://www.dsr.inpe.br/ canasat>. Acesso em 28 jan. 11.

11 JOUSSIER, D.; CATROUX, G. Mise au point d'un milleau de culture pour le denombrement de Beauveria tenella dans le sols. Entomophaga, v. 21, n. 3, p. 223-225, 1976.

12 LANZA, L.M.; MONTEIRO, A.C.; MALHEIROS, E.B. População de Metarhizium anisopliae em diferentes tipos e graus de compactação do solo. Ciência Rural, v. 34, n. 6, p. 1757-1762, 2004.

13 LANZA, L.M.; MONTEIRO, A.C.; MALHEIROS, E.B. Sensibilidade de Metarhizium anisopliae à temperatura e umidade em três tipos de solos. Ciência Rural, v. 39, n. 1, p. 6-12, 2009.

14 LEITE, L.G.; MACHADO, L.A.; AGUILLERA, M.M.; RODRIGUES, R.C.D.; NEGRISOLI JUNIOR, A.S. Patogenicidade de Steinernema e Heterorhabditis (Nematoda: Rhabditida) contra ninfas da cigarrinha-das-raízes da cana-de-açúcar, Mahanarva fimbriolata (Hemiptera: Cercopidae). Agricultura, v. 78, n. 1, p. 139-148, 2003.

15 LEITE, G.H.P.; CRUSCIOL, C.A.C.; SILVA, M. de A.; VENTURINI FILHO, W.G. Maturadores e qualidade tecnológica da cana-de-açúcar variedade RB855453 em início de safra. Bragantia, v. 68, n. 3, p. 781-787, 2009.

16 LOUREIRO, E. de S.; MOINO JUNIOR, A.; ARNOSTI, A.; SOUZA, G.C. de. Efeito de produtos fitossanitários químicos utilizados em alface e crisântemo sobre fungos entomopatogênicos. Neotropical Entomology, v. 31, n.2, p. 263-269, 2002.

$17 \mathrm{MOCHI}$, D.A.; MONTEIRO, A.C.; BARBOSA, J.C. Action of pesticides to Metarhizium anisopliae in soil. Neotropical Entomology, v. 34, n. 6, p. 961-971, 2005.

18 RODRIGUES, B.N.; ALMEIDA, F.S. Guia de herbicidas. 5. ed. Londrina: lapar, 2005. 591 p.

19 SANTOS, T.M.C.; MONTEIRO, R.T.R. Número de microrganismos e atividade da urease na presença de aldicarbe e endosulfan no solo. Scientia Agrícola, v. 51, n. 1, p. 123-130, 1994.

20 TAMAI, M.A.; ALVES, S.B.; LOPES, R.B.; FAION, M.; PADULLA, L.F.L. Toxicidade de produtos fitossanitários para Beauveria bassiana (Bals.) Vuill. Arquivos do Instituto Biológico, v. 69, n.3, p. 89-96, 2002.

21 TIXIER, C.; BOGAERTS, P.; SANCELME, M.; BONNEMOY, F.; TWAGILIMANA, L.; CUER, A.; BOHATIER, J.; VESCHANBRE, $\mathrm{H}$. Fungal biodegradation of a phenylurea herbicide, diuron: structure and toxicity of metabolites. Pest Management Science, v. 56, n. 5, p. 455-462, 2000.

22 TÓTOLA, M.R.; CHAER, G.M. Microrganismos e processos microbiológicos como indicadores da qualidade do solo. In: ALVAREZ VENEGAS, V.H.; SCHAEFER, C.E.G.R.; BARROS, N.F. DE; MELLO, J.W.V. DE; COSTA, L.M. DA. (Eds.). Tópicos em ciência do solo. Viçosa: Sociedade Brasileira de Ciência do Solo, 2002. p. 195-276.

23 VIANA, R.S.; MUTTON, M.A.; BARBOSA, V.; DURIGAN, A.M.P.R. Maturadores químicos na cana-de-açúcar (Saccharum spp) aplicados em final de safra. Revista de Biologia e Ciência da Terra, v. 7, n.2, p. 100-107, 2007.

24 ZILLI, J.E.; RUMJANEK, N.G.; XAVIER, G.R.; COUTINHO, H.L.C.; NEVES, M.C.P. Diversidade microbiana como indicador de qualidade do solo. Cadernos de Ciência e Tecnologia, v. 20, n. 3, p. 391-411, 2003.

\section{AGRADECIMENTOS}

À Coordenadoria de Aperfeiçoamento de Pessoal de Ensino Superior (CAPES) pela concessão da bolsa de mestrado à primeira autora. 